\title{
A comparative analysis of forest cover and catchment water yield relationships in northern China
}

\author{
Shuai Wang ${ }^{\mathrm{a}}$, Bo-Jie Fu ${ }^{\mathrm{a}, *}$, Chan-Sheng $\mathrm{He}^{\mathrm{b}}$, Ge Sun ${ }^{\mathrm{c}}$, Guang-Yao Gao ${ }^{\mathrm{a}}$ \\ a State Key Laboratory of Urban and Regional Ecology, Research Center for Eco-Environmental Sciences, Chinese Academy of Sciences, P.O. Box 2871, Beijing 100085, PR China \\ ${ }^{\mathrm{b}}$ Department of Geography, Western Michigan University, Kalamazoo, MI 49008-5054, USA \\ ' USDA-Forest Service, Southern Research Station, 920 Main Campus Dr., Venture Center 2, Suite 300, Raleigh, NC 27606, USA
}

\section{A R T I C L E I N F O}

\section{Article history:}

Received 15 March 2011

Received in revised form 28 May 2011

Accepted 6 June 2011

Available online 7 July 2011

\section{Keywords:}

Forest cover

Runoff coefficient

Water yield

Northern China

Evapotranspiration

\begin{abstract}
A B S T R A C T
During the past few decades, China has implemented several large-scale forestation programs that have increased forest cover from $16.0 \%$ in the 1980 s to $20.4 \%$ in 2009 . In northern China, water is the most sensitive and limiting ecological factor. Understanding the dynamic interactions between forest ecosystems and water in different regions is essential for maximizing forest ecosystem services. We examined forest cover and runoff relationships in northern China using published data from a variety of sources. In the Loess Plateau region, forest cover is not correlated with annual precipitation $(r=0.08, p>0.05)$ at micro $\left(<50 \mathrm{~km}^{2}\right)$ and meso scales $\left(50-1000 \mathrm{~km}^{2}\right)$, while they are positively correlated at macro $\left(>1000 \mathrm{~km}^{2}\right)$ scale $(r=0.77, p<0.05)$. Moreover, forest cover is negatively correlated with the runoff coefficient $(r=-0.64, p<0.05)$. In Northwest China, natural forest distribution is highly correlated with annual precipitation $(r=0.48, p<0.05)$ but not with the runoff coefficient $(r=-0.09, p>0.05)$. In Northeast China, we found a positive relationship between forest cover and the runoff coefficient $(r=0.77, p<0.05)$, but the correlation between forest cover and precipitation was not significant $(r=0.28, p>0.05)$. The multiple stepwise regression analysis indicated that runoff was influenced by altitude, annual precipitation, forest cover, and PET (potential evapotranspiration) in Northeast China. We concluded that geographic differences could mask the true role of forests in the partitioning of rainfall into runoff and evapotranspiration (ET) in a catchment. In determining the forest-water relationship, one must consider climatic controls on ET in addition to forest cover. Forests could potentially enhance the complementary relationship between ET and PET. Therefore, a greater amount of ET in forested areas may decrease the PET on a regional scale. (c) 2011 Elsevier B.V. All rights reserved.
\end{abstract}

\section{Introduction}

Forests provide important ecosystem services and have long been recognized as playing an important role in environmental rehabilitation, biodiversity maintenance, carbon sequestration, bio-fuel, timber production, amenities, and social benefits (Calder, 2007). However, forests cannot usually simultaneously produce multiple, positive ecosystem services because of the trade-offs among different or competing functions. Maximizing one service may cause substantial declines of other services (Bennett et al., 2009). As the demand for reliable provisions from almost all ecosystems including forests is increasing globally (Millennium Ecosystem Assessment, 2005), it is essential to understand the dynamic relationships among all forest ecosystem services. Water is the most sensitive and limiting ecological factor in forest systems. Forests and water supplies are closely linked, and trade-off between water and biological carbon sequestration has been recognized (Jackson

\footnotetext{
* Corresponding author. Tel./fax: +86 1062923557.

E-mail address: bfu@rcees.ac.cn (B.-J. Fu).
}

et al., 2005; Sun et al., 2006, 2007). Therefore, maximizing the production of forest ecosystem services must essentially understand the interactions between forests and water at the regional scale.

In China, forests have long been recognized for their role in environmental protection and the development of human societies (Sun et al., 2008). During the past few decades, in addition to changes in forest management principles and strategies, China has implemented several large-scale forestation programs that have increased forest cover (the proportion of forest to total land area) from $16.0 \%$ in the 1980s to $20.4 \%$ in 2009 (State Forestry Administration, 2009). Forestation programs include reforestation (forest regenerations from original forest land) and afforestation (forest establishment in non-forest land) practices. The earliest large scale forestation program in China is the Three-North Forest Shelterbelt Program, which began in 1978 and will end in 2050. The program is underway in Northeast, North Central, and Northwest China, together referred to as the Three-North Plains. The program, covering a total of 590 counties in 17 arid and semiarid provinces and autonomous regions, was designed to improve forest cover in arid and semiarid China from $5 \%$ in the 1980 s to $15 \%$ by 2050 to combat desertification and 
control dust storms. This project is being implemented in three stages (1978-2000, 2001-2020, and 2021-2050) and follows eight engineering plans (2002-2010) (Wang et al., 2010). Following the severe flood in 1998 in the Yangtze River Basin, another forestation program, the Natural Forest Protection Program (NFPP) was conducted in late 1998 in 17 provinces and autonomous regions along the upper reaches of the Yangtze River and the upper and middle reaches of the Yellow River. The program area contains 73 million ha of natural forests, which amount to $69 \%$ of the total natural forests area in China (Zhang et al., 2000). A third program, "Sloping Land Conversion Program" or "Grain for Green" was launched in 1999 to return cultivated land with slopes of $25^{\circ}$ or more to perennial vegetation across China. The target of this conversion was 32 million ha by 2010 , and the goal of the program is to reduce water and soil erosion by increasing forest cover. The program has been implemented in more than 2000 counties across 25 provinces and autonomous regions (Wei et al., 2008). Implementation of above three large-scale forest restoration programs has generated a significant growth in forest resources. The forest cover increased by 20.5 million ha since 2003, and the extent of China's total forest plantations is approximately 54 million ha, which accounts for one fourth of the world's total forested area (Raloff, 2009).

Forests can strongly influence the partitioning of precipitation into ET (evapotranspiration, which includes evaporation and transpiration) and runoff in a catchment (Schymanski et al., 2009; Wang et al., 2011). The change in soil water and groundwater storage is assumed to be negligible over years at catchment scale (Wang et al., 2011). The water balance equation can be written as:

$P=\mathrm{ET}+R$

where $P$ is precipitation or the water input for a catchment, ET is evapotranspiration or the amount of water lost to the atmosphere from a catchment, and $R$ is surface runoff measured as stream flow, often referred to as the water yield of a catchment. The runoff coefficient, which equals $R / P$, indicates the proportion of a catchment's water yield from precipitation. Forests may influence precipitation patterns at local to regional scales by changing surface-atmosphere transfers of heat and moisture. However, it is proven that this effect is very small (van Dijk and Keenan, 2007). Therefore, the effect of forests on catchment water yield can be attributed mainly to the changes in ET throughout the year (Farley et al., 2005; Jackson et al., 2005; Wang et al., 2011).

Over the past century, significant progress has been made in understanding the relationship between forests and water, especially in North America, Europe, and Australia (Andréassian, 2004). Paired catchment studies have provided a basis for assessing relationships between catchment vegetation, ET and runoff (Peel, 2009). Hibbert (1967) reviewed results from 39 paired catchment experiments and concluded that a reduction in forest cover increased the water yield, while the establishment of forest cover on sparsely vegetated land decreased the water yield. Bosch and Hewlett (1982) reviewed 94 paired experiments, and reported a diminished uncertainty and quantified trends for different vegetation manipulations. Brown et al. (2005) analyzed 72 additional paired catchment studies with respect to the study of Bosch and Hewlett (1982), increasing the total datasets of the paired catchment experiment to 166. The authors grouped the experiments into four broad categories: afforestation (conversion of short vegetation to forest), deforestation (conversion of forest to short vegetation), regrowth (forest removal and regrowth), and forest conversion (replacement of one forest type with another). Their review also considered long-term annual changes, adjustment time scales, the seasonal pattern of flows, and changes in both annual and seasonal flow duration curves. These reviews indicated that harvesting forests generally increased annual water yield and low flows, and had a great impact on small peak flows but a minor impact on large peak flows. In general, forestation practices decreased annual water yields and low flows due to increases in ET, but it had minimal or no effect on floods, particularly for large floods (Hornbeck et al., 1993; Stednick, 1996; Sahin and Hall, 1996; Andréassian, 2004). However, the hydrologic recovery processes after forestation are not simply the reversal of those of deforestation. Deforestation experiments generally reach a new equilibrium faster than forestation experiments (Brown et al., 2005). Zhao et al. (2009a) also illustrated that water yields changed following vegetation changes and this process occurred not only in small catchments but also in larger ones. Wei et al. (2008) stated that forestation campaigns were not likely to lead to large scale changes in annual water yield, low flow, or flood peaks before the hydrologic properties of degraded soils were fully improved.

China is characterized by diverse climatic and topographic conditions that sustain various forest ecosystems ranging from boreal forests in the North to tropical rain forests in the South. Many hydrologic studies in China's forests have focused on a single process, including forest canopy interception, stemflow, throughfall, and ET at the field level (Zhang and Yu, 1988; Zhou et al., 1994; Zhang et al., 2001, 2004; Liu et al., 1996, 2003). Surprisingly, there are few established standard paired catchment experiments (Wei et al., 2008). Hence, empirical observations and the limited data available on the environmental influences of forests are often inconclusive and even contradictory, especially concerning hydrologic cycles (Wei et al., 2003, 2005). This situation can likely be attributed to the relatively late inception of studies on the forest-water relationship in the 1980s and the highly diverse hydrologic processes that exist within a wide range of geographic and climatic regions in China (Sun et al., 2008). Sun et al. (2006) suggested that the large spatial and temporal variability of hydrologic responses to reforestation will follow gradients in climate, topography, soil, and disturbances in China. The different responses will depend on several key factors including climate, soil conditions, and stage of vegetation recovery.

Understanding of the hydrologic effects of forestation is especially critical in the semi-arid Loess Plateau and other areas of northern China that have been experiencing chronic water shortages. The trade-offs between reforestation and water resources are likely most significant in northern China (Sun et al., 2006). Although numerous studies have been conducted in individual regions, a comparative cross-region synthesis on forest-water relationships is still needed. The objectives of this study are as follows. First is to review studies on forest and water interactions. Second is to discuss the relationships between forest cover and runoff coefficients in the Loess Plateau region, Northwest China, and Northeast China. Third is to characterize the regional differences in forest-water patterns.

\section{Methods}

\subsection{Data compilation}

We compiled catchment datasets from published in peer-reviewed Chinese and international journals. We examined data from catchments where forest cover and runoff were measured. The final datasets used for this analysis contained 70 catchments for Loess Plateau, 26 ones for Northwest, and 22 ones for Northeast China. The Qingshui catchment of Loess Plateau underwent three distinguished forest cover stages (1960-1969, 1970-1979, and 1980-1989). Each stage was considered as a different catchment in this study. Therefore, the total actual datasets for Loess Plateau is 72. The detailed description of the complied catchments datasets is shown in Table 1. We analyzed the datasets for two main variables, forest cover and runoff coefficients. 
Table 1

Descriptions of the compiled catchments.

\begin{tabular}{|c|c|c|c|c|c|c|c|c|c|c|c|c|c|}
\hline Name & Site & Data period & $\begin{array}{l}\text { Catchment } \\
\text { area }\left(\mathrm{km}^{2}\right)\end{array}$ & $\begin{array}{l}\text { Altitude } \\
(\mathrm{m})\end{array}$ & $\begin{array}{l}\text { River } \\
\text { slope } \\
(\% o)\end{array}$ & $\begin{array}{l}\text { Forest } \\
\text { cover } \\
(\%)\end{array}$ & $\begin{array}{l}\text { Average } \\
\text { annual } \\
\text { temperature } \\
\left({ }^{\circ} \mathrm{C}\right)\end{array}$ & $\begin{array}{l}\text { July average } \\
\text { temperature } \\
\left({ }^{\circ} \mathrm{C}\right)\end{array}$ & $\begin{array}{l}\text { Average } \\
\text { annual } \\
\text { precipitation } \\
(\mathrm{mm})\end{array}$ & $\begin{array}{l}\text { Average } \\
\text { annual } \\
\text { PET } \\
(\mathrm{mm})\end{array}$ & $\begin{array}{l}\text { Average } \\
\text { annual } \\
\text { runoff } \\
(\mathrm{mm})\end{array}$ & $\begin{array}{l}\text { Runoff } \\
\text { coefficient }\end{array}$ & Source \\
\hline \multicolumn{14}{|l|}{ Northeast China } \\
\hline Ashen river & Maoershan & 1971-1987 & 183 & 450 & 7.1 & 50 & 3.03 & 22.27 & 638 & 1195 & 205.6 & 0.32 & (1) \\
\hline Ashen river & Acheng & 1971-1987 & 2313 & 190 & 1.2 & 35 & 3.25 & 22.38 & 626 & 1296 & 148.2 & 0.24 & (1) \\
\hline Douzuizi & Binxian & 1971-1987 & 99 & 400 & 9.1 & 70 & 3.65 & 22.7 & 536 & 1003 & 167.8 & 0.31 & (1) \\
\hline Mayi river & Lianhua & 1971-1987 & 8664 & 310 & 1.5 & 66 & 2.4 & 21.8 & 633 & 1159 & 191.8 & 0.3 & (1) \\
\hline Woken river & Woken & 1971-1987 & 4164 & 200 & 0.5 & 59 & 3.23 & 21.87 & 510 & 1240 & 118.5 & 0.23 & (1) \\
\hline Hengdao & Hengdaohe & 1971-1987 & 145 & 750 & 15.4 & 80 & 3.3 & 22.03 & 719 & 898 & 371.5 & 0.52 & (1) \\
\hline Anbang river & Fulitun & 1971-1987 & 579 & 90 & 5.3 & 71 & 3.45 & 22.1 & 535 & 1284 & 168.1 & 0.31 & (1) \\
\hline Naoli river & Caizuizi & 1971-1987 & 20796 & 100 & 0.3 & 44 & 2.5 & 21.45 & 509 & 1169 & 92.8 & 0.18 & (1) \\
\hline Nianzi river & Boli & 1971-1987 & 142 & 350 & 12 & 70 & 3.5 & 22 & 541 & 1099 & 113.2 & 0.21 & (1) \\
\hline Nenjiang & Kumotun & 1971-1987 & 31693 & 410 & 1.4 & 52 & -2.18 & 19.03 & 487 & 1045 & 169.5 & 0.35 & (1) \\
\hline Namoer & Dedu & 1971-1987 & 7200 & 350 & 7.3 & 34 & 1.45 & 21.02 & 520 & 1088 & 69.2 & 0.13 & (1) \\
\hline Wuyuerhe & Beian & 1971-1987 & 2592 & 300 & 0.7 & 40 & 0.2 & 20.7 & 547 & 1132 & 145.1 & 0.27 & (1) \\
\hline Wuyuerhe & Yian & 1971-1987 & 7423 & 220 & 0.6 & 22 & 1 & 21.23 & 495 & 1229 & 85.1 & 0.17 & (1) \\
\hline Nenjiang & Fulaerji & 1971-1987 & 123190 & 350 & 0.1 & 49 & 0.42 & 20.76 & 466 & 1203 & 140.8 & 0.3 & (1) \\
\hline Neijiang & Jiangqiao & 1971-1987 & 177253 & 260 & 0 & 33 & 1.9 & 21.84 & 459 & 1221 & 115.4 & 0.25 & (1) \\
\hline $\mathrm{Ni}$ river & Nihe & $>10$ years & 617 & 180 & 1.6 & 0 & 2.25 & 22.25 & 521 & 1537 & 65.4 & 0.13 & (2) \\
\hline Hulan river & Lanxi & $>10$ years & 27305 & 190 & 0.6 & 35 & 1.79 & 21.89 & 559 & 1185 & 135.4 & 0.24 & (3) \\
\hline Tangwang & Chenming & $>10$ years & 18857 & 420 & 1 & 94 & -0.4 & 20.47 & 599 & 989 & 264.7 & 0.44 & (3) \\
\hline Yongcui & Dailing & $>10$ years & 169 & 580 & 19.7 & 90 & -0.05 & 20.25 & 588 & 1000 & 260.4 & 0.44 & (2) \\
\hline Wutong river & Baoquanling & $>10$ years & 2750 & 350 & 4.2 & 50 & 1.6 & 21 & 564 & 1000 & 193.9 & 0.34 & (2) \\
\hline Emuer river & & $>10$ years & 15523 & & & 63.7 & & & 418 & & 150.3 & 0.36 & (4) \\
\hline Pangu river & & $>10$ years & 4369 & & & 68.7 & & & 433 & & 212.5 & 0.49 & (4) \\
\hline \multicolumn{14}{|l|}{ Northwest China } \\
\hline Yili river & Yamadu & 1960-1973 & 44516 & 2050 & & 4.7 & 9.5 & 24 & 269 & 985 & 240.4 & 0.89 & (5) \\
\hline Piliqing & Piliqing & 1960-1973 & 794 & 1820 & & 5.7 & 9.9 & 24.1 & 432 & 1440 & 224.7 & 0.52 & (5) \\
\hline Boertala & Wenquan & 1960-1973 & 2206 & 2938 & & 5.6 & 3.5 & 19.9 & 205 & 852 & 147.5 & 0.72 & (5) \\
\hline Manasi river & Kensiwate & 1960-1973 & 4637 & 3260 & & 3 & 6.3 & 24.4 & 349 & 1003 & 251.3 & 0.72 & (5) \\
\hline Taxi river & Shimenzi & $1960-1973$ & 664 & 3170 & & 7.2 & 4.4 & 20.6 & 433 & 868 & 348.6 & 0.81 & (5) \\
\hline Hutubi river & Shimen & 1960-1973 & 1840 & 3250 & & 6 & 5.6 & 21 & 389 & 918.7 & 230.2 & 0.59 & (5) \\
\hline Santun river & Nianpanzhuang & 1960-1973 & 1636 & 2791 & & 7.2 & 6.1 & 23 & 253 & 879 & 220.1 & 0.87 & (5) \\
\hline Toutun river & Zhicaichang & 1960-1973 & 840 & 3024 & & 19.3 & 4.8 & 19.8 & 396 & 825 & 273.8 & 0.69 & (5) \\
\hline Wulumuqi & Yingxiongqiao & 1960-1973 & 924 & 3310 & & 4.7 & 1.7 & 15.1 & 477 & 661 & 263.5 & 0.55 & (5) \\
\hline Kaiken river & Kaikenhe & 1960-1973 & 371 & 3500 & & 7.5 & 3.2 & 17.6 & 608 & 894 & 259.8 & 0.43 & (5) \\
\hline Mulei river & Yuejin shuiku & 1960-1973 & 467 & 3150 & & 14.3 & 4.3 & 20.6 & 434 & 1071 & 85.2 & 0.2 & (5) \\
\hline Yiwu river & Weizixia & 1960-1973 & 1057 & 2590 & & 2.7 & 7.5 & 25.4 & 60 & 1509 & 51.8 & 0.86 & (5) \\
\hline Sidelong & & 1973-1980 & 110 & & 0.1 & 32 & & & 560 & & 413.6 & 0.74 & (6) \\
\hline Tianlaochi & & 1973-1980 & 13 & & 0.1 & 65.9 & & & 599 & & 380.8 & 0.64 & (6) \\
\hline Upper heihe & & 1973-1980 & 2557 & & & 5.9 & & & 37 & & 15.9 & 0.43 & (6) \\
\hline Kukesu & & 1957-1962 & 5379 & 1210 & & 4.6 & & & 450 & 944 & 410.1 & 0.91 & (7) \\
\hline Gongnaisi & & 1957-1962 & 4123 & 900 & & 13.3 & & & 466 & 873 & 351 & 0.75 & (7) \\
\hline Qiedeke & & 1957-1962 & 291 & 940 & & 11.7 & & & 459 & 942 & 436.4 & 0.95 & (7) \\
\hline Jiagesitai & & 1985-1989 & 231 & 1410 & & 21.7 & & & 341 & 983 & 251.1 & 0.74 & (7) \\
\hline Wuertakeleisi & & 1985-1989 & 938 & 1330 & & 6.8 & & & 233 & 778 & 154.6 & 0.66 & (7) \\
\hline Kusumuqieke & & 1985-1989 & 1103 & 1400 & & 16.3 & & & 240 & 800 & 91.5 & 0.38 & (7) \\
\hline Bayingou & & 1985-1989 & 1579 & 850 & & 3.3 & & & 244 & 923 & 193.8 & 0.8 & (7) \\
\hline Jingou river & & 1985-1989 & 1273 & 810 & & 6.2 & & & 237 & 1278 & 246.7 & 1.04 & (7) \\
\hline Hongnigou & & 1985-1989 & 3902 & 1100 & & 2.5 & & & 348 & 1107 & 251.4 & 0.72 & (7) \\
\hline Baiyang river & & 1985-1989 & 252 & 1100 & & 8 & & & 252 & 897 & 242.1 & 0.96 & (7) \\
\hline Xidalongkou & & 1985-1989 & 255 & 1200 & & 13.4 & & & 284 & 915 & 172.5 & 0.61 & (7) \\
\hline \multicolumn{14}{|l|}{ Loess Plateau } \\
\hline Beipo & & 2001-2005 & 2 & & & 92.5 & & & 357 & & 1.8 & 0.01 & (8) \\
\hline Liugou & & 2001-2005 & 2 & & & 83.4 & & & 360 & & 5.4 & 0.02 & (8) \\
\hline Nanbeiyao & & 2001-2005 & 1 & & & 0 & & & 358 & & 27.5 & 0.08 & (8) \\
\hline Liujiaao & & $2002-2005$ & 4 & & & 82.7 & & & 369 & & 5.9 & 0.02 & (8) \\
\hline Jingou & & $2002-2005$ & 3 & & & 15.2 & & & 367 & & 16.9 & 0.05 & (8) \\
\hline Chashang & & 2002-2005 & 32 & & & 70 & & & 375 & & 4.5 & 0.01 & (9) \\
\hline Diantou & & $2001-2005$ & 34 & & & 56 & & & 560 & & 11.2 & 0.02 & (9) \\
\hline Wannianbao & & 2001-2005 & 286 & & & 80 & & & 333 & & 1 & 0 & (9) \\
\hline Beizhangdian & & $>5$ years & 270 & & & 17 & & & 438 & & 3.5 & 0.01 & (9) \\
\hline Siping & & $>5$ years & 192 & & & 9 & & & 407 & & 5.7 & 0.01 & (9) \\
\hline Nanguan & & $>5$ years & 257 & & & 52 & & & 455 & & 5 & 0.01 & (9) \\
\hline Gedonggou & & $>5$ years & 73 & & & 63 & & & 350 & & 2.8 & 0.01 & (9) \\
\hline Lengkou & & $>5$ years & 76 & & & 90 & & & 580 & & 11.6 & 0.02 & (9) \\
\hline Xiaodian & Gansu & $>10$ years & 272 & & & 15 & & & 531 & & 47 & 0.09 & (10) \\
\hline Caijiaomiao & Gansu & $>10$ years & 270 & & & 15 & & & 530 & & 32 & 0.06 & (10) \\
\hline Yaofenggou & Gansu & $>10$ years & 219 & & & 20 & & & 511 & & 40 & 0.08 & (10) \\
\hline Hejiapo & Gansu & $>10$ years & 100 & & & 20 & & & 489 & & 30 & 0.06 & (10) \\
\hline Nanxiao & Gansu & 1959-1962 & 28 & & & 0 & & & 500 & & 12 & 0.02 & (11) \\
\hline Wangjia & Gansu & 1959-1962 & 48 & & & 90 & & & 639 & & 10 & 0.02 & (11) \\
\hline
\end{tabular}


Table 1 (continued)

\begin{tabular}{|c|c|c|c|c|c|c|c|c|c|c|c|c|}
\hline Name & Site & Data period & $\begin{array}{ll}\text { Catchment } & \text { Altitude } \\
\text { area }\left(\mathrm{km}^{2}\right) & (\mathrm{m})\end{array}$ & $\begin{array}{l}\text { River } \\
\text { slope } \\
(\%)\end{array}$ & $\begin{array}{l}\text { Forest } \\
\text { cover } \\
(\%)\end{array}$ & $\begin{array}{l}\text { Average } \\
\text { annual } \\
\text { temperature } \\
\left({ }^{\circ} \mathrm{C}\right)\end{array}$ & $\begin{array}{l}\text { July average } \\
\text { temperature } \\
\left({ }^{\circ} \mathrm{C}\right)\end{array}$ & $\begin{array}{l}\text { Average } \\
\text { annual } \\
\text { precipitation } \\
(\mathrm{mm})\end{array}$ & $\begin{array}{l}\text { Average } \\
\text { annual } \\
\text { PET } \\
(\mathrm{mm})\end{array}$ & $\begin{array}{l}\text { Average } \\
\text { annual } \\
\text { runoff } \\
(\mathrm{mm})\end{array}$ & $\begin{array}{l}\text { Runoff } \\
\text { coefficient }\end{array}$ & Source \\
\hline Qingjian & Zichang & 1951-1963 & 916 & & 0 & & & 509 & & 34 & 0.07 & (11) \\
\hline Xiangu & Anminggou & 1951-1963 & 24 & & 0 & & & 624 & & 37 & 0.06 & $(12)$ \\
\hline Beiluo & Liujiahe & 1951-1963 & 7315 & & 18.3 & & & 475 & & 29 & 0.06 & $(12)$ \\
\hline Fengchuan & Linzhen & 1951-1963 & 1121 & & 94.4 & & & 555 & & 18 & 0.03 & $(12)$ \\
\hline Beiluo & Zahngcunyi & 1951-1963 & 5400 & & 97 & & & 568 & & 19 & 0.03 & $(12)$ \\
\hline Xiangu & Hongmiaogou & 1951-1963 & 42 & & 98.5 & & & 636 & & 29 & 0.05 & $(12)$ \\
\hline Xi Qingshui & & 1963-1981 & 706 & & 4.1 & & & 439 & & 36 & 0.08 & (13) \\
\hline Dong Qingshui & & 1963-1982 & 775 & & 39.8 & & & 500 & & 44 & 0.09 & (13) \\
\hline Qingshui & Shanxi & 1960-1969 & 435 & & 25.3 & & & 589 & & 55 & 0.09 & $(14)$ \\
\hline Qingshui & Shanxi & 1970-1979 & 435 & & 55.3 & & & 551 & & 46 & 0.08 & (14) \\
\hline Qingshui & Shanxi & 1980-1989 & 435 & & 57.9 & & & 516 & & 23 & 0.05 & (14) \\
\hline Yan & Ganguyi & 1959-1970 & 5981 & & 8 & & & 536 & & 42 & 0.08 & $(15)$ \\
\hline Liujia & Beiluohe & 1959-1970 & 7325 & & 9 & & & 462 & & 38 & 0.08 & (15) \\
\hline Xinshui & Daning & 1959-1970 & 3992 & & 10 & & & 527 & & 50 & 0.1 & $(15)$ \\
\hline Zhouchuan & Jiaxian & 1959-1970 & 436 & & 10 & & & 436 & & 53 & 0.12 & $(15)$ \\
\hline Hulu & Zhangcunji & 1959-1970 & 4715 & & 100 & & & 569 & & 29 & 0.05 & $(15)$ \\
\hline Fengchun & Linzhen & 1959-1970 & 1121 & & 100 & & & 539 & & 23 & 0.04 & $(15)$ \\
\hline Wudinghe & Dingshi & 1980-2000 & 327 & & 0 & & & 375 & & 36 & 0.1 & $(15)$ \\
\hline Wudinghe & Hanjiamao & $1980-2000$ & 2452 & & 0 & & & 317 & & 31 & 0.1 & $(15)$ \\
\hline Huangpuchuan & Huangpuchuan & 1980-2000 & 3175 & & 0 & & & 366 & & 33 & 0.09 & $(15)$ \\
\hline Wudinghe & Hengshan & 1980-2000 & 2415 & & 0 & & & 378 & & 21 & 0.06 & $(15)$ \\
\hline Wudinghe & Lijiahe & $1980-2000$ & 8.7 & & 0.1 & & & 392 & & 31 & 0.08 & $(15)$ \\
\hline Wudinghe & Caoping & 1980-2000 & 187 & & 0.1 & & & 403 & & 38 & 0.09 & $(15)$ \\
\hline Wudinghe & Mahuyu & 1980-2000 & 371 & & 0.1 & & & 391 & & 38 & 0.1 & (15) \\
\hline Kuyehe & Wangdaohengta & $1980-2000$ & 3839 & & 0.2 & & & 346 & & 40 & 0.12 & $(15)$ \\
\hline Jialuhe & Shenjiawan & 1980-2000 & 1121 & & 0.6 & & & 386 & & 38 & 0.1 & $(15)$ \\
\hline Wudinghe & Qingyangcha & 1980-2000 & 662 & & 0.6 & & & 413 & & 34 & 0.08 & $(15)$ \\
\hline Kuyehe & Xinmiao & 1980-2000 & 1527 & & 0.8 & & & 357 & & 53 & 0.15 & $(15)$ \\
\hline Kuyehe & Shenmu & $1980-2000$ & 7298 & & 0.9 & & & 356 & & 55 & 0.15 & $(15)$ \\
\hline Kuyehe & Weijiachuan & 1980-2000 & 8645 & & 0.9 & & & 361 & & 56 & 0.16 & $(15)$ \\
\hline Gushanchuan & Gaoshiya & 1980-2000 & 1263 & & 1 & & & 385 & & 41 & 0.11 & $(15)$ \\
\hline Wudinghe & Dingjiagou & 1980-2000 & 23422 & & 1 & & & 348 & & 33 & 0.1 & $(15)$ \\
\hline Wudinghe & Baijiachuan & $1980-2000$ & 29662 & & 1.1 & & & 362 & & 33 & 0.09 & $(15)$ \\
\hline Wudinghe & Zhaoshiku & 1980-2000 & 15325 & & 1.3 & & & 342 & & 29 & 0.09 & $(15)$ \\
\hline Yanhe & Ansai & 1980-2000 & 1334 & & 3.8 & & & 446 & & 40 & 0.09 & $(15)$ \\
\hline Qingjianhe & Zichang & 1980-2000 & 913 & & 4 & & & 444 & & 41 & 0.09 & $(15)$ \\
\hline Yanhe & Yanan & 1980-2000 & 3208 & & 4.2 & & & 456 & & 39 & 0.09 & $(15)$ \\
\hline Qingliangshigou & Yangjiapo & $1980-2000$ & 283 & & 4.3 & & & 431 & & 32 & 0.07 & (15) \\
\hline Xianchuanhe & Jiuxian & 1980-2000 & 1562 & & 4.4 & & & 412 & & 11 & 0.03 & $(15)$ \\
\hline Qingjianhe & Yanchuan & $1980-2000$ & 3468 & & 5 & & & 455 & & 39 & 0.09 & $(15)$ \\
\hline Quchanhe & Peigou & $1980-2000$ & 1023 & & 6.4 & & & 478 & & 26 & 0.05 & $(15)$ \\
\hline Yanhe & Xinghe & $1980-2000$ & 479 & & 7.7 & & & 439 & & 37 & 0.08 & $(15)$ \\
\hline Yanhe & Ganguyi & 1980-2000 & 5891 & & 9.7 & & & 470 & & 34 & 0.07 & $(15)$ \\
\hline Zhujiachuan & Xialiuji & $1980-2000$ & 2881 & & 10.3 & & & 424 & & 11 & 0.03 & $(15)$ \\
\hline Qiushuihe & Linjiaping & $1980-2000$ & 1873 & & 11 & & & 448 & & 25 & 0.06 & $(15)$ \\
\hline Sanchuanhe & Houdacheng & $1980-2000$ & 4102 & & 21.1 & & & 471 & & 43 & 0.09 & (15) \\
\hline Yanhe & Zaoyuan & 1980-2000 & 719 & & 24.8 & & & 488 & & 35 & 0.07 & (15) \\
\hline Xinshuihe & Daning & $1980-2000$ & 3992 & & 28.2 & & & 484 & & 23 & 0.05 & (15) \\
\hline Weifenhe & Xinxian & $1980-2000$ & 650 & & 34 & & & 446 & & 28 & 0.06 & (15) \\
\hline Zhouchuanhe & Jixian & 1980-2000 & 436 & & 37.9 & & & 493 & & 21 & 0.04 & (15) \\
\hline Yunyanhe & Xinshihe & 1980-2000 & 1662 & & 48 & & & 507 & & 20 & 0.04 & $(15)$ \\
\hline Yunyanhe & Linzhen & $1980-2000$ & 1121 & & 65.3 & & & 508 & & 16 & 0.03 & (15) \\
\hline Shiwanghe & Dacun & 1980-2000 & 2141 & & 72.7 & & & 528 & & 28 & 0.05 & $(15)$ \\
\hline
\end{tabular}

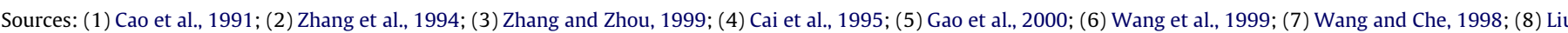

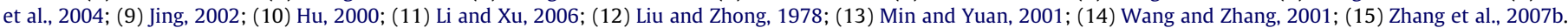

\subsection{Comparative analysis}

Our goal was to determine the relationship between forest cover and catchment water yields and to compare regional differences of this relationship. First, we correlated forest cover and precipitation and then performed a correlation and regression analysis between forest cover and the runoff coefficient to remove the impact of precipitation on water yields. Subsequently, we conducted a multiple stepwise regression analysis on runoff, runoff coefficient and PET, with respect to forest cover, annual precipitation, altitude and annual temperature variables using SPSS for Windows 13.0.

\section{Study areas}

The catchments used in this study are located in northern China, including the Loess Plateau, Northwest and Northeast China (Fig. 1). Each region is described below.

\subsection{The Loess Plateau}

The Loess Plateau of China is located in the upper and middle reaches of the Yellow River between the western Taihang 


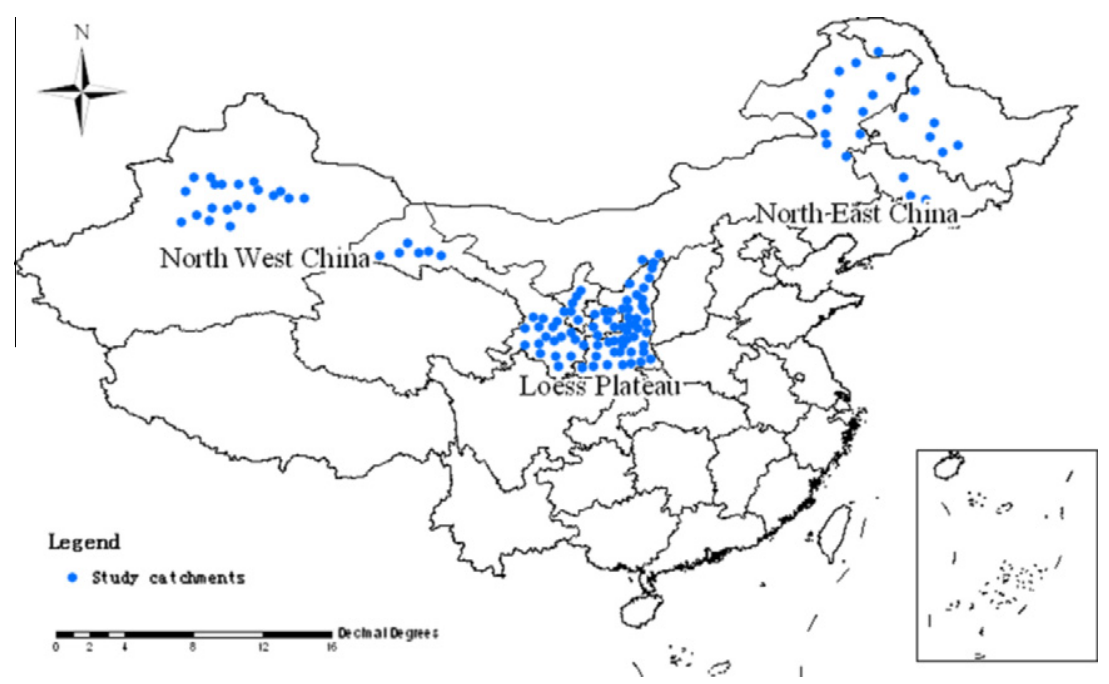

Fig. 1. The study catchments in the Loess Plateau, Northwest China and Northeast China.

Mountains, eastern Riyue-Helan Mountains, northern Qinling Mountains, and southern Yinshan Mountains. The primary implementation area of the "Sloping Land Conversion Program" stretches across a southern semi-humid forest zone, a middle semi-arid forest-steppe zone, and a northern semi-arid drought-prone typical steppe zone. Common forest species include black locust (Robinia pseudoacia), Chinese pine (Pinus tabulaeformis Carr.), apple (Malus domestica Borkh), Littleleaf peashrub (Caragana microphylla) and Seabuckthorn shrub (Hippophae rhammoides) in agricultural forest plantations. However, due to water shortage, these trees grow quite slowly, appearing "small but old" (Li et al., 2008; McVicar et al., 2007).

\subsection{Northwest China}

Northwest China is characterized by little precipitation and high potential evapotranspiration (PET). We chose the Qilian and Tianshan Mountains as study areas. Both of these areas contain complex ecosystems consisting of forests, grasslands and glaciers. These areas are typical arid ecosystems with typical continental climates (Zhao et al., 2009b).

\subsection{Northeast China}

The northeast region extends over ten degree latitudes from south to north and includes territories of Liaoning, Jilin, Heilongjiang and the eastern part of the Inner Mongolia Autonomous Region. The region is characterized by a temperate climate and has one of the lowest ET rates in China (Ni and Zhang, 2000). Forest types in this area include cold-temperate conifer mixed forests, temperate conifer and broadleaf mixed forests, and warm-temperate deciduous broadleaf mixed forests. Plantation and secondary forests account for a large proportion of the total forested area (Liu et al., 1996). Much of the catchment forest and runoff data used in this paper were from the Songhuajiang and Heilongjiang basins.

\section{Results}

\subsection{The Loess Plateau}

In the Loess Plateau region, the correlation analysis revealed that there was no statistically significant relationship between forest cover and precipitation $(r=0.08, p>0.05)$ at micro $\left(<50 \mathrm{~km}^{2}\right)$ and meso scales $\left(50-1000 \mathrm{~km}^{2}\right)$, while they were positively correlated at macro $\left(>1000 \mathrm{~km}^{2}\right)$ scale $(r=0.77, p<0.05)$ (Fig. 2$)$. The analysis of 72 average annual catchment values of forest cover and runoff coefficient in the Loess Plateau indicated that there was a significant negative relationship between these two variables $(r=-0.64, p<0.05$, Fig. 2$)$.

\subsection{Northwest China}

In Northwest China, forests are generally not planted, and natural forests are distributed within areas with higher precipitation. We analyzed data from published articles, including 26 average annual catchment values of forest cover and precipitation in the northern Tianshan Mountains and Qilian Mountains. The results showed that forest cover and precipitation are significantly correlated $(r=0.48, p<0.05$, Fig. 3 ). Forest cover and runoff coefficients in the two regions also exhibited a negative, but not statistically significant trend $(r=-0.09, p>0.05$, Fig. 3$)$.

\subsection{Northeast China}

In Northeast China, analysis of 22 average annual catchment values of forest cover and runoff coefficient showed that there was a positive relationship between the two $(r=0.77, p<0.05$, Fig. 4). However, the correlation between forest cover and precipitation was not significant $(r=0.28, p>0.05$, Fig. 4). Because the datasets from Northeast China were more complete, we also conducted a stepwise regression analysis to examine key influences on runoff.

$R=-203.8+1.17 \mathrm{FC}+0.45 P+0.17 A\left(r^{2}=0.86, p<0.05\right)$

where $R$ is runoff, $A$ is altitude, $P$ is precipitation, FC is forest cover.

Forest cover, precipitation, and altitude explain $10 \%, 15 \%$, and $62 \%$ of the variability of $R$, respectively.

$\mathrm{PET}=1432.4-0.45 A-2.69 \mathrm{FC}\left(r^{2}=0.68, p<0.05\right)$

where PET is potential evapotranspiration calculated by standard Pan evaporation at the nearby weather stations.

Altitude and forest cover explain $55 \%$ and $11 \%$ of the variations of PET, respectively.

Linear stepwise regression analyses suggested that runoff was dependent on altitude, precipitation, and forest cover, while PET was dependent on altitude and forest cover in Northeast China. 

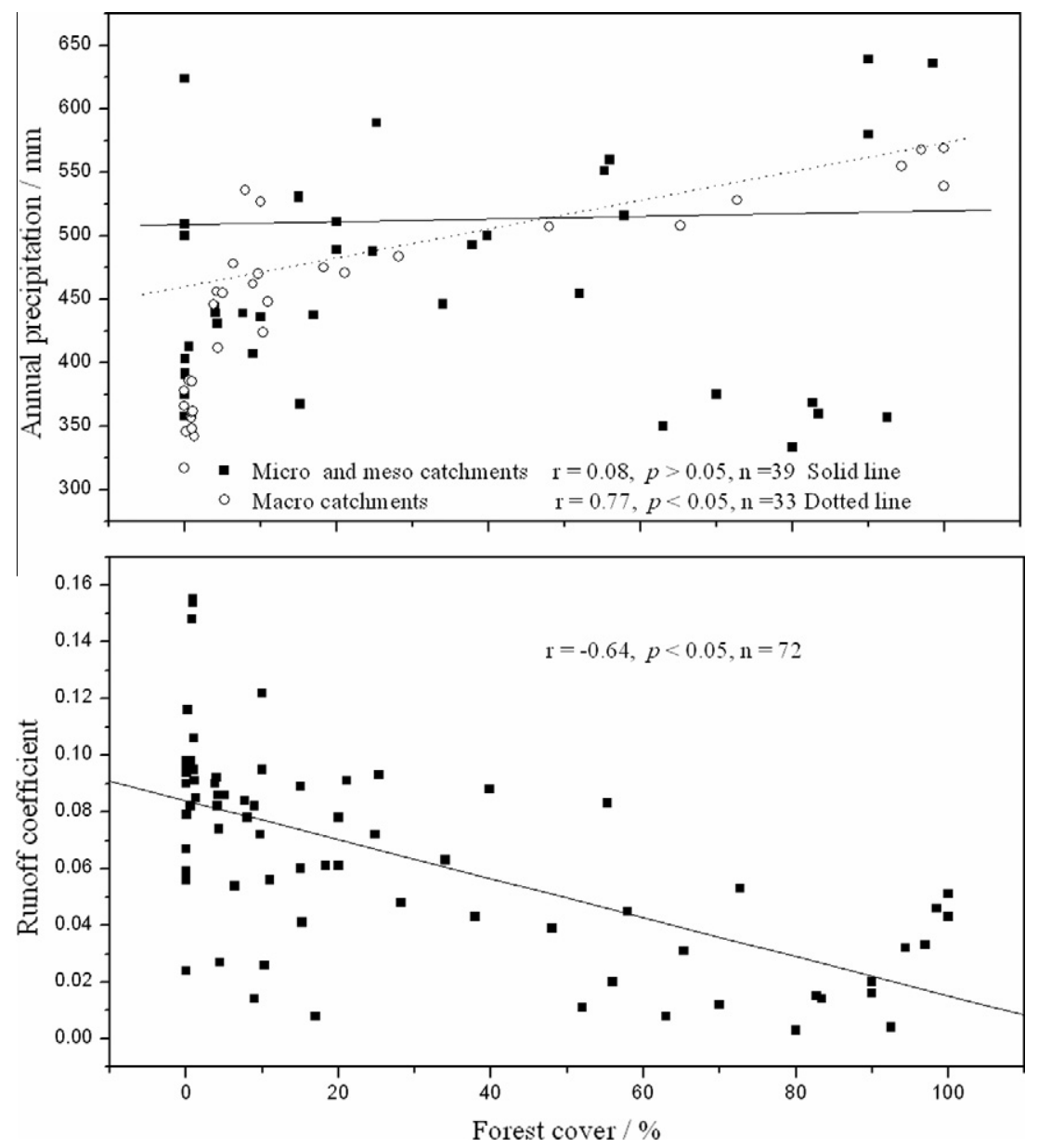

Fig. 2. The relationship between forest cover, annual precipitation and runoff coefficients in the Loess Plateau.

\section{Discussion}

\subsection{Relationships between forest cover and catchment water yield}

The results indicated that the correlations between forest cover and precipitation was geographically variable and dependent on the scale (Wang et al., 2011). For example, at Loess Plateau, they showed no significant correlation at micro and meso scales. However, at macro scale they showed a significant positive correlation. This fact that inequalities exist between levels of forest cover and precipitation is likely related to decisions about how to manage forestation programs and not dependent upon precipitation. These inequalities may also contribute to geographical variations in the relationship between forest cover and runoff coefficients. Chang and Wang (2005) calculated the average of annual overland flow reduction after forestation was $7.7 \mathrm{~mm}$ at the plot scale. Huang et al. (2003) reported that this reduction was $4.6 \mathrm{~mm}$ at the paired catchments about $1 \mathrm{~km}^{2}$. However, about $50 \mathrm{~mm}$ reduction was determined mainly based on climatic conditions by Sun et al. (2006). The main processes and influence factors changed with scales. Although the magnitudes of the effects of forests are different, the trends are consistent at both small and large scales. With the increase of catchment area, runoff coefficient also increased $(r=0.32, p<0.05$, Table 1$)$ in the Loess Plateau (the figure not shown here), but no obvious trend was found in the other two regions because their samples are not enough.

The stage of forests development also affected water yield (Farley et al., 2005). Zhang et al. (2006) reported an increasing annual ET of plantations with increasing tree age. We deliberately used the average values of the records of longer duration (range of 3-21 years) in an attempt to minimize these errors. This approach has already been shown to be a simple and effective one which can assess the hydrological effects of forestation (Wang et al., 2011). If the forests coverage showed dramatically change, we analyzed separately. For example, we distinguished Qingshui basin at Loess Plateau as three stages: 1960-1969, 1970-1979, 1980-1989. Large scale afforestation in China began in the 1980s. It seems that the forests in these catchments have not yet attained their climatic effects so far.

The results for the Loess Plateau were consistent with the general conclusions of the global paired catchment studies. Studies conducted in the Loess Plateau by Liu and Zhong (1978) more than 30 years ago also found that forested catchments on loess soils had lower water yield amounts ( $25 \mathrm{~mm} / \mathrm{yr}$.) and that the runoff coefficient was less than that of adjacent non-forested areas. The authors also estimated that forests in the Loess Plateau region may reduce the annual water yield by $37 \%$. Based on continental-scale simulations using a generalized ET model, Sun et al. (2006) concluded that an average water yield reduction may vary from about $50 \mathrm{~mm} / \mathrm{yr}$ (50\%) in the semi-arid Loess Plateau region to about $300 \mathrm{~mm} / \mathrm{yr}(30 \%)$ in the tropical southern region. Other studies (Lu et al., 2009; Liu et al., 2005; Zhang et al., 2006; Li, 2001; Huang and Liu, 2002) have shown that there was an obvious decline in water yield as forest cover increased. However, many of these studies observed increased flow in low flow seasons, indicating that forested catchments produced greater base flows and more natural springs.

In Northwest China, the Tianshan Mountain north hill and the Qilian Mountains are complex ecosystems consisting of forests, grassland and glaciers. The relationships between forest cover 

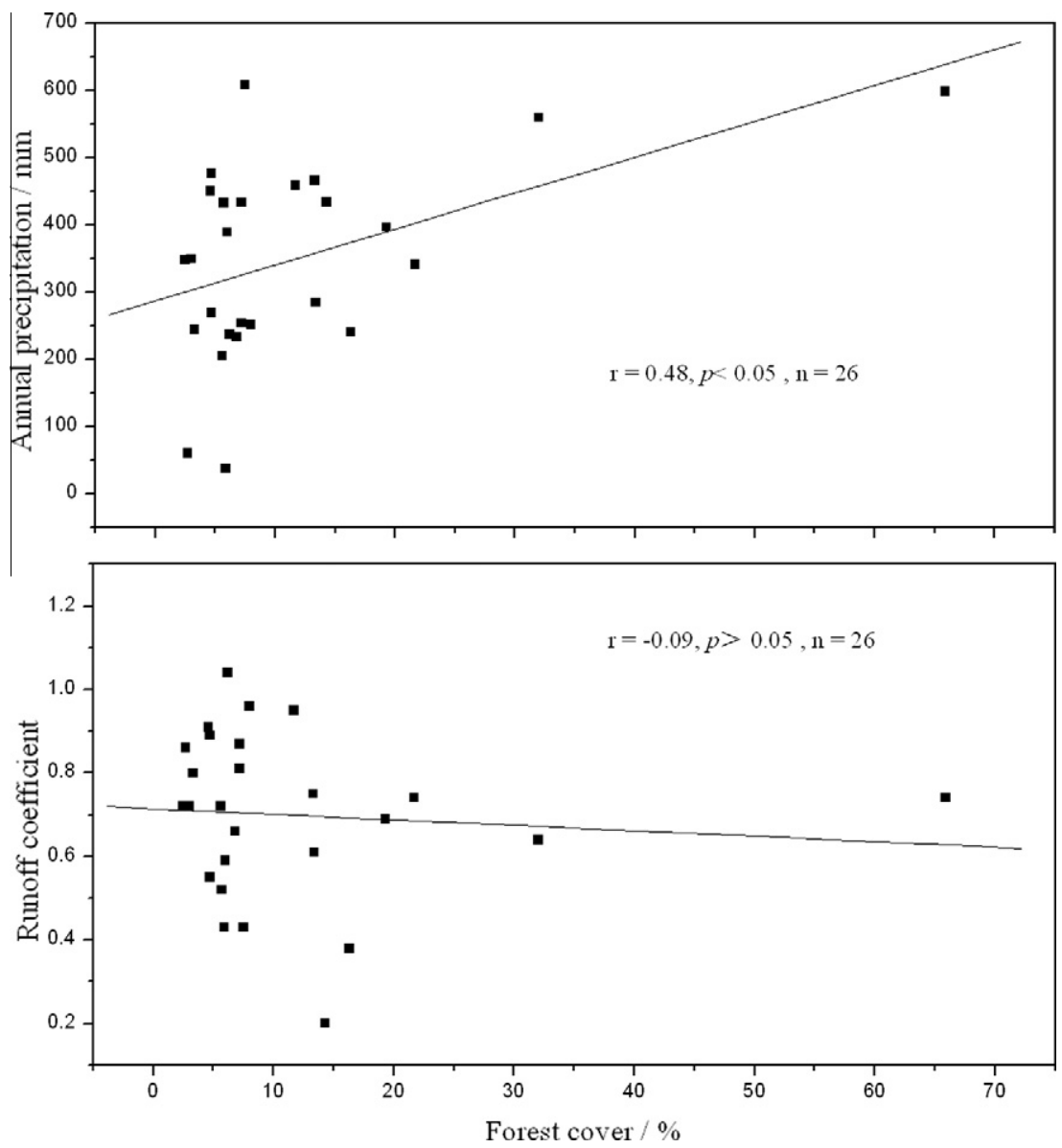

Fig. 3. The relationship between forest cover, annual precipitation and runoff coefficients in Northwest China.

and water yield are equally as complex. Many of these catchments occur in less than $30 \%$ forest cover, and few occur in areas with more than $60 \%$ cover. The results showed a negative but not statistically significant relationship between forest cover and runoff coefficient for Northwest China. In the Tianshan Mountain north hill region, if forest cover percentage was $0-10 \%$, the water yield increased with forest cover percentage, but when forest cover percentage was greater than $10 \%$, the runoff coefficient decreased as forest cover percentage increased (Gao et al., 2000). Long-term observations by the local research station on Qilian Mountain indicated that forest vegetation might reduce surface runoff, postpone runoff period, and minimize flooding (Wang and Che, 1998; Wang et al., 1999).

Although our studies found results similar to an earlier study by Cao et al. (1991), specifically a positive relationship between forest cover and water yield for northeastern China, we believe that ET is significantly influenced by PET and elevation in addition to precipitation. In contrast, in the arid Loess Plateau region, ET is largely controlled by precipitation and vegetation. Previous studies in northern China on the relationships between forest cover and water yield have been conflicting (Chen and Li, 2001). In fact, we observed that forests tend to be distributed at higher altitudes $(r=0.56, p<0.05)$, with steeper slopes $(r=0.71, p<0.05)$, more precipitation $(r=0.55, p<0.05)$ and lower PET values $(r=-0.74$, $p<0.05$ ) (Table 1 ). Therefore, these regions partition more precipitation into runoff, making the runoff coefficient higher.

Observed relationships between forest cover and water yield in different regions are complex and can be inconsistent. Wei et al. (2003) attributed the inconsistencies to several factors: (1) large, heterogeneous basins have a large buffering capacity and may mask the effects of forest cover; (2) different measurement methods are used with varying biases and errors; and (3) differences in climate and watershed characteristics among the contrasting basins may obscure the effects of forest cover. While differences exist among the three regions, in Northeast China, forest cover positively correlated with catchment water yield, but in the Loess Plateau and Northwest China a negative correlation was observed, while the correlation were not significant for Northwest China.

\subsection{Regional differences}

ET has a large impact on the balance of water within an ecosystem. The water yield from a catchment is altered through changes in transpiration, interception, and evaporation. Zhang (2001) postulated that the long-term average annual ET of a catchment depends upon the minimum value of potential evaporation (PET) and the available water (AW) for evapotranspiration within the catchment. Forestation may affect these two values. Forestation increases the value of AW through transpiration, or by removing water from soil and intercepting precipitation. Contrarily, forestation reduces the value of PET for several possible reasons. First, forestation increased near infrared reflectance but caused no significant differences in visible radiation reflectance compared with other land cover, resulting in a reduced absorption of net radiation (Cheng and Chen, 2004). Second, accumulated ET resulted in low temperature and high humidity, further reducing PET (Qiu et al., 1999). Lastly, fog intercepted by the forest canopy constituted a significant portion of total precipitation, but this water was not recorded as precipitation and ultimately evaporates (Clus et al., 2008). Throughout this process, it will consume a great deal 

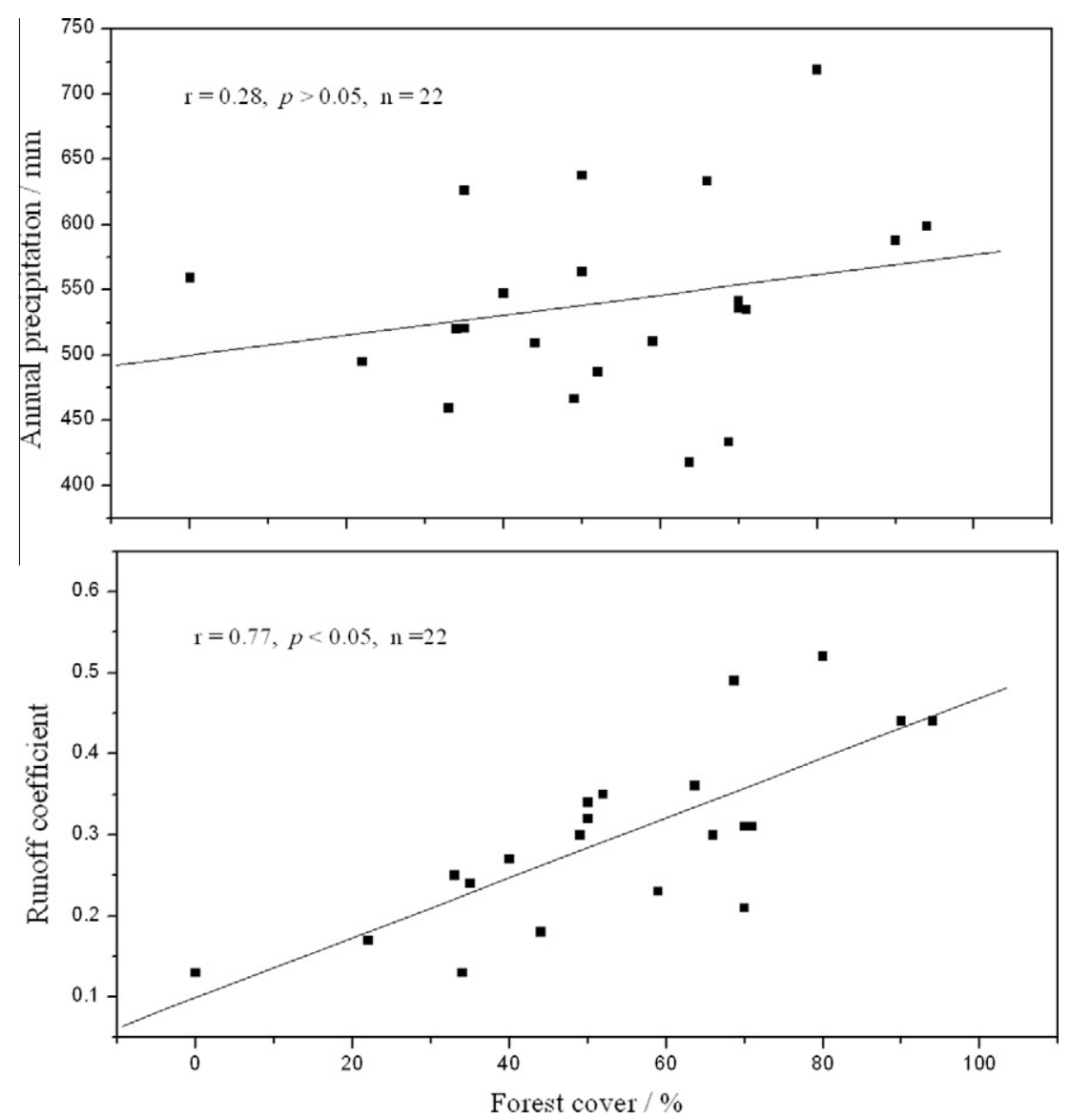

Fig. 4. The relationship between forest cover, annual precipitation and runoff coefficients in Northeast China.

of the PET (Kalthoff et al., 2006). Therefore, the "fog drip" precipitation in forests will greatly reduce PET (Beysens et al., 2007). These findings agree with the complementary relationship between ET and PET (Xu and Singh, 2005). The influence of forestation on water yield can be illustrated in two extreme cases. In humid conditions, the value of AW is greater than PET and actual ET depends on the value of PET. Therefore, when a forestation program is implemented, the value of PET will decrease and the loss of water will be smaller, which resulting the increase of water yield of the catchment. In arid conditions where the value of AW is less than the PET value, actual ET depends on the value of AW, and forestation alteration will therefore increase the value of AW and actual ET, reducing the amount of water yield.

Forestation activities may prompt land conversion in one of these two extreme cases, but the ultimate result depends on changes in AW and PET, which are determined by local geographic conditions. The Loess Plateau area experiences an arid to semi-arid climate, and the PET is far greater than available water, so the actual ET is controlled by AW. When forestation was implemented in this extremely arid region, AW increased while PET decreased, and actual ET increased correspondingly. Subsequently, water yield decreased in the Loess Plateau area. In dry Northwest China, the situation is similar, but water is available in the form of glacier runoff. Therefore, the mechanism is more complex and a change in the proportion of forest cover has little impact on water yield in dry Northwest China. However, in the low-temperature, high-humidity environments of the Northeast, "fog drip," or "horizontal precipitation" occurs. Fog is not recorded as precipitation, but the cumulative amount increased by forest cover may be greater than the amount of increased ET, so larger forested areas result in increased water yields. This analysis is consistent with other low-temperature and high-humidity areas such as the upper reaches of Yangtze River and Russia (Sun et al. 2007; Liu et al., 2007; Zhang et al., 2007a; Ma and Zhang, 1998). This has also been confirmed by a recent study by Zhou et al. (2010), which illustrated that large-scale forest recovery did result in a water yield increase over the past 50 years in the humid Guangdong Province.

Catchments are integrated systems, and impacts on water yield should be considered collectively rather than focusing on specific processes such as interception, stem flow, and infiltration. The main water input into ecosystems is precipitation, including vertical precipitation (the most commonly recognized and measured form of precipitation) and horizontal precipitation (not recorded as precipitation). The key outputs are ET and runoff (water yield). Influence of forests on global precipitation patterns is complex and at local to regional scales it is evidenced to be very small (van Dijk and Keenan, 2007). Therefore, the change in water yield is mainly determined by the difference between the increase in horizontal precipitation and actual ET. However, "fog drip" is difficult to quantify and is therefore rarely reported. Many studies have focused on calculating ET, but the accuracy of their results is still difficult to determine. Some models estimate variations in ET based on vegetation changes to compare the impacts of vegetation changes on ET, thus lacking well-thought out field verifications. Therefore, the precise calculation of ET is still a priority for forest hydrology.

\subsection{Potential limitations}

Various sources of uncertainty were consistently flagged in the compiled datasets and throughout the analyses. Few causal correlations exist between confounding variables, a fact likely related to the heterogeneity of the datasets. In addition, the stage of forest 
development could not be precisely included in the analysis because these data were not reported in many studies. Average values of the longer duration records were used in an attempt to minimize these errors. The analysis also includes catchments with very different spatial scales, scale effects on precipitation and runoff coefficient were discussed. Finally, the pattern of rainfall may relate to the species of trees, which may influence the relationship between forest cover and runoff. Because of dataset limitations, these issues were necessarily overlooked. Despite these limitations, this comparative analysis of forest-water relations is useful and can potentially contribute to the understanding of forest hydrology in different regions of northern China.

\section{Conclusions}

Water shortages are common in northern China, and large-scale forestation efforts must consider the impact of increased forest cover on hydrological processes. ET is the major source of consumption in a catchment, and changes in water yield depend on variations in ET caused by increased or decreased forest cover. This study concluded that geographic differences could mask the true role of forests in partitioning rainfall into runoff and ET in a catchment. Future studies must consider climatic controls on ET in addition to forest cover when determining the forest-water relationship. Our hypothesis is that trees can increase the water available for ET and enhance the complementary relationship between actual ET and PET such that more actual ET will lower the PET. In arid conditions, the principal control on evaporation is the availability of plantwater, while under wet conditions, the main control is atmospheric demand, which is determined by PET. Under intermediate conditions, the control depends upon the relative importance of each of these factors. In northern China, the Loess Plateau is relatively dry, Northeast China is relatively wet, and Northwest China presents a complex system based on glacier runoff. An analysis of these ecosystems may not be able to pinpoint a cause-effect relationship but can confirm that there is a relationship between them. However, the data used in this study are incomplete and cover a short period of time. Long-term observations are needed to gain a better understanding of the forest-water interactions in these three regions.

\section{Acknowledgments}

This research was supported by the National Basic Research Program of China (No. 2009CB421104), the National Natural Science Foundation of China (No. 40930528), the External Cooperation Program of the Chinese Academy of Sciences (No. GJHZ0948) and the CAS/SAFEA International Partnership Program for Creative Research Teams of "Ecosystem Processes and Services." We thank the anonymous reviewers for their detailed and constructive comments which greatly improve the quality of the manuscript.

\section{References}

Andréassian, V., 2004. Waters and forests: from historical controversy to scientific debate. J. Hydrol. 291, 1-27.

Bennett, E.M., Peterson, G.D., Gordon, L., 2009. Understanding relationships among multiple ecosystem services. Ecol. Lett. 12, 1394-1404.

Beysens, D., Clus, O., Mileta, M., Milimouk, I., Muselli, M., Nikolayev, V.S., 2007. Collecting dew as a water source on small islands: the dew equipment for water project in Bisevo (Croatia). Energy 32, 1032-1037.

Bosch, J.M., Hewlett, J.D., 1982. A review of catchment experiments to determine the effect of vegetation changes on water yield and evapotranspiration. J. Hydrol. 55, 3-23.

Brown, A.E., Zhang, L., McMahon, T.A., Western, A.W., Vertessy, R.A., 2005. A review of paired catchment studies for determining changes in water yield resulting from alterations in vegetation. J. Hydrol. 310, 28-61.

Cai, T.J., Zhou, X.F., Yang, W.H., 1995. The effects of forest fire on streamflow in Daxinganling. Sci. Sil. Sin. 31 (5), 403-408 (in Chinese with English abstract).
Calder, I.R., 2007. Forests and water - ensuring forest benefits outweigh water costs. For. Ecol. Manage. 251, 110-120.

Cao, Y., Li, Q., Cai, T., Shen, Y., 1991. The effects of forest coverage on river flow in the Songhua catchments. In: Zhou, X. (Ed.), Studies on Forest Ecosystems. Northeast Forestry University Press, Harbin, pp. 396-402 (in Chinese).

Chang, D.D., Wang, L.X., 2005. Influences of artificial afforestation on annual runoff amount in upper and middle reaches of Yellow River. J. Mount. Sci. 23 (6), 678686 (in Chinese with English abstract).

Chen, J.F., Li, X.B., 2001. The impact of forest change on watershed hydrology. J. Nat. Resour. 5, 474-481 (in Chinese with English abstract).

Cheng, G.W., Chen, G.R., 2004. Study on the controlled factors of watershed evapotranspiration and its regional difference under the effect of forest change. J. Mount. Res. 2, 175-178 (in Chinese with English abstract).

Clus, O., Ortega, P., Muselli, M., Milimouk, I., Beysens, D., 2008. Dew formation and collection in a humid tropical climate (French Polynesia). J. Hydrol. 361, 159171

Farley, K.A., Jobbagy, E.G., Jackson, R.B., 2005. Effects of afforestation on water yield: a global synthesis with implications for policy. Global Change Biol. 11, 15651576. doi:10.1111/j.1365-2486.2005.01011.x.

Gao, X.H., Pan, C.D., Li, J.G., 2000. A study on relation between the Tianshan Mountains north sloping hill land forest and potamic runoff. J. Xinjiang Agric. Univ. 1, 25-29 (in Chinese with English abstract).

Hibbert, A.R., 1967. Forest treatment effects on water yield. In: Sopper, W.E., Lull, H.W. (Eds.), International Symposium on Forest Hydrology. Pergamon, Oxford, pp. 527-543.

Hornbeck, J.W., Adams, M.B., Corbett, E.S., Verry, E.S., Lynch, J.A., 1993. Long-term impacts of forest treatments on water yield: a summary for northeastern USA. J. Hydrol. 150 (2/4), 323-344.

$\mathrm{Hu}$, X.L., 2000. Influence of forest cover on the water resources in Loess Hill Region of Gansu Province. Advan. Water Resour. 11 (2), 199-202 (in Chinese with English abstract).

Huang, M.B., Liu, X.Z., 2002. Regulation effect of forest vegetation on watershed runoff in the Loess Plateau. Chin. J. Appl. Ecol. 9, 1057-1060 (in Chinese with English abstract).

Huang, M.B., Zhang, L., Gallichand, J., 2003. Runoff responses to afforestation in a watershed of the Loess Plateau, China. Hydrol. Process. 17 (13), 2599-2609. doi:10.1002/hyp.1454

Jackson, R.B., Jobbagy, E.G., Avissar, R., Roy, S.B., Barrett, D.J., Cook, C.W., Kathleen, A., Farley, K.A., le Maitre, D.C., Bruce, A., McCarl, B.A., Murray, B.C., 2005. Trading water for carbon with biological sequestration. Science 310, 1944-1947.

Jing, L., 2002. Research on the effect of the forest cover on sediment and runoff in Shaanxi province. Northwest Water Resour. Engin. 13 (3), 53-57 (in Chinese with English abstract).

Kalthoff, N., Fiebig-Wittmaack, M., Meibner, C., Kohler, M., Uriarte, M., BischoffGaub, I., Gonzales, E., 2006. The energy balance, evapo-transpiration and nocturnal dew deposition of an arid valley in the Andes. J. Arid Environ. 65, $420-443$.

Li, Y.S., 2001. Effects of forest on water circle on the Loess Plateau. J. Nat. Resour. 5, 427-432 (in Chinese with English abstract).

Li, G.Y., Xu, X.X., 2006. Rediscussion about the effects of forests on precipitation and annual runoff. J. Northwest For. Univ. 26 (1), 1-611 (in Chinese with English abstract).

Li, J., Chen, B., Li, X.F., Zhao, Y.J., Jiang, B., Hu, W., Cheng, J., Shao, M.A., 2008. Effects of deep soil desiccation on artificial forestlands in different vegetation zones on the Loess Plateau of China. ACTA Ecol. Sin. 28 (4), 1429-1445 (in Chinese with English abstract).

Liu, C.M., Zhong, J., 1978. Effects of forests on annual streamflow in the Loess Plateau region. ACTA Ecol. Sin. 33 (1), 12-126 (in Chinese with English abstract).

Liu, S.R., Wen, Y.G., Wang, B., Zhou, G.Y., 1996. Ecohydrological functions of forest ecosystems in China. China Forestry Publication House (in Chinese), Beijing.

Liu, S.R., Sun, P.S., Wen, Y.G., 2003. Comparative analysis of hydrological functions of major forest ecosystems in China. ACTA Phytoeco. Sin. 27 (1), 16-22 (in Chinese with English abstract)

Liu, H.F., Zhu, Q.F., Wei, T.X., 2004. Impact of forests on runoff in small watershed in western Shanxi of Loess Plateau. J. Soil Water Conserv. 18 (2), 5-10, in Chinese with English abstract.

Liu, H.F., Zhu, Q.K., Wei, T.X, 2005. Effects of forests on the runoff of small watershed in Loess Plateau in western Shanxi. J. Arid Land Resour. Environ. 5 61-66 (in Chinese with English abstract).

Liu, S.R., Sun, P.S., Wang, J.X., Chen, L.W., 2007. Hydrological functions of forest vegetation in upper reaches of the Yangtze River. J. Nat. Resour. 16 (5), 451-457 (in Chinese with English abstract).

Lu, A.G., Suo, A.N., Pang, D.Q., Wang, T.M., 2009. Effect of forest on catchments hydrological process in middle Loess Plateau. Res. Soil Water Conserv. 16 (4), 15, in Chinese with English abstract.

Ma, L.Q., Zhang, Y.R., 1998. A preliminary study on the forest hydrology effect in Chongqing Area, Southwestern China. J. Beijing For. Univ. 20 (1), 14-20 (in Chinese with English abstract).

McVicar, T.R., Li, L.T., Van Niel, T.G., Zhang, L., Li, R., Yang, Q.K., Zhang, X.P., Mu, X.M., Wen, Z.M., Liu, W.Z., Zhao, Y.A., Liu, Z.H., Gao, P., 2007. Developing a decision support tool for China's re-vegetation program: simulating regional impacts of afforestation on average annual streamflow in the Loess Plateau. For. Ecol. Manage. 251, 65-81.

Millennium Ecosystem Assessment, 2005. Ecosystems and Human well-being: Synthesis. Island Press, Washington, DC. 
Min, Q.W., Yuan, J.Z., 2001. Effects of forest on regional precipitation: results from some different analyses and their comparisons. J. Nat. Resour. 16 (5), 467-473 (in Chinese with English abstract).

Ni, J., Zhang, X.S., 2000. Climate variability, ecological gradient and the Northeast China Transect (NECT). J. Arid Environ. 46, 313-325.

Peel, M.C., 2009. Hydrology: catchment vegetation and runoff. Progr. Phys. Geogr. $12,1-8$

Qiu, G.Y., Kazuro, M., Tomohisa, Y., Robert, J.L., 1999. Experimental verification of a mechanistic model to partition evapotranspiration into soil water and plant evaporation. Agric. For. Meteorol. 93, 79-93.

Raloff, J. 2009. China defends its reputation. Sciencenews. http://www.sciencenews. org/view/generic/id/51055/title/Climate_China_defends_its_reputation/.

Sahin, V., Hall, M.J., 1996. The effects of afforestation and deforestation on water yields. J. Hydrol. 178 (1/4), 293-309.

Schymanski, S.J., Sivapalan, M., Roderick, M.L., Hutley, L.B., Beringer, J., 2009. An optimality-based model of the dynamic feedbacks between natural vegetation and the water balance. Water Resour. Res. 45, W01412. doi:10.1029/ 2008WR006841.

State Forestry Administration, 2009. http://www.forestry.gov.cn/.

Stednick, J.D., 1996. Monitoring the effects of timber harvest on annual water yield. J. Hydrol. 176 (1/4), 79-95.

Sun, G., Zhou, G.Y., Zhang, Z.Q., Wei, X.H., McNulty, S.G., Vose, J.M., 2006. Potential water yield reduction due to reforestation across China. J. Hydrol. 328, 548-558.

Sun, G., Zhou, G.Y., Zhang, Z.Q., Wei, X.H., McNulty, S.G., Vose, J.M., 2007. Forest and water relations: hydrologic implications to forestation campaigns in China. In: Jin, W. (Ed.), Wetland and Water Resource Modeling and Assessment: A Watershed Perspective. CRC Press, pp. 71-88.

Sun, G., Liu, S.R., Zhang, Z.Q., Wei, X.H., 2008. Forest hydrology in China: introduction to the featured collection. JAWRA 44 (5), 1073-1075. doi:10.1111/j.1752-1688.2008.00248.x.

van Dijk, A.I.J.M., Keenan, R.J., 2007. Planted forests and water in perspective. For. Ecol. Manage. 251, 1-9. doi:10.1016/j.foreco.2007.06.010.

Wang, J.Y., Che, K.J., 1998. Runoff produced laws of complex forest watershed in Qilian Mountain. J. Soil Water Conserv. 1, 23-28 (in Chinese with English abstract).

Wang, L.X., Zhang, Z.Q., 2001. Impacts of forest vegetation on watershed runoff in dryland areas. J. Nat. Resour. 16 (5), 439-444 (in Chinese with English abstract).

Wang, J.Y., Che, K.J., Yan, K.L., Wang, Y.L., He, H.Y., 1999. Analysis of the runoff components in the forestry areas of the Qilian Mountains and their temporal and spatial variation. J. glacia. geocry. 1, 59-63 (in Chinese with English abstract).

Wang, X.M., Zhang, X.C., Hasi, E., Dong, Z.B., 2010. Has the Three Norths Forest Shelterbelt Program solved the desertification and dust storm problems in arid and semiarid China? J. Arid. Environ. 74 (1), 13-22.

Wang, Y.H., Yu, P.T., Feger, K., Wei, X.H., Sun, G., Bonell, M., Xiong, W., Zhang, S., Xu, L., 2011. Annual runoff and evapotranspiration of forestlands and nonforestlands in selected basins of the Loess Plateau of China. Ecohydrology 4, 277-287.

Wei, X.H., Zhou, X.F., Wang, C.K., 2003. Impacts of the temperate forests on hydrology, Northeast of China. For. Chron. 79, 297-300.
Wei, X.H., Liu, S.R., Zhou, G.Y., Wang, C., 2005. Hydrological processes of key Chinese forests. Hydrol. Process. 19 (1), 63-75.

Wei, X.H., Sun, G., Liu, S.R., Jiang, H., Zhou, G.Y., Dai, L.M., 2008. The foreststreamflow relationship in China: a 40-years retrospect. JAWRA 44 (5), 1076 1085.

Xu, C.Y., Singh, V.P., 2005. Evaluation of three complementary relationship evapotranspiration models by water balance approach to estimate actual regional evapotranspiration in different climatic regions. J. Hydrol. 308, 105121.

Zhang, Z.Q., 2001. Forest vegetation impacts on the runoff generation: a review. J Nat. Resour. 16 (1), 79-84, in Chinese with English abstract.

Zhang, Z.Z., Yu, X.X., 1988. The major progress on Chinese forest hydrological research. J. Beijing For. Univ. 10 (2), 79-87 (in Chinese with English abstract).

Zhang, Q.F., Zhou, X.F., 1999. Influence of forest on runoff discharges in Tangwang River and Hulan River basins of Heilongjiang province. J. Plant Resour. Environ. 8 (1), 22-27 (in Chinese with English abstract).

Zhang, Q.F., Zhou, X.F., Cai, T.J., 1994. Influence of forest on snowflow in the middle region of Heilongjiang province. J. Plant Resour. Environ. 3, 36-40 (in Chinese with English abstract).

Zhang, P.C., Shao, G.F., Zhao, G., Dennis, C.L.M., Parker, G.R., Dunning, J.B., Li, Q.L., 2000. China's forest policy for the 21 st century. Science 288 (5474) 2135-2136.

Zhang, L., Dawes, W.R., Walker, G.R., 2001. Response of mean annual evapotranspiration to vegetation changes at catchment scale. Water Resour. Res. 37 (3), 701-708.

Zhang, Z.Q., Wang, L.X., Wang, S.P., 2004. Forest hydrology research in China. Sci. Soil Water Conserv. 2 (2), 68-73, in English with Chinese abstract.

Zhang, X.M., Yu, X.X., Wu, S.H., Liu, H.F., Zhang, X.P., Wei, T.X., 2006. Influence of forest vegetation on runoff and sediment transport of watershed in loess area. Sci. Soil Water Conserv. 3, 48-53 (in Chinese with English abstract).

Zhang, H.J., Cheng, J.H., Chen, Z.W., 2007a. Effects of forest variety on runoff and sediment in the Three-Gorge Region of Yangtze River. Res. Soil Water Conserv. 14 (1), 1-3 (in Chinese with English abstract).

Zhang, X.P., Zhang, L., Mu, X.M., Li, R., 2007b. The mean annual water balance in the Hekou-Longmen Section of the middle Yellow River:Testing of the regional scale water balance model and its calibration. ACTA Geog. Sin. 62 (7), 753-763 (in Chinese with English abstract).

Zhao, F.F., Zhang, L., Xu, Z.X., 2009a. Effects of vegetation cover change on streamflow at a range of spatial scales. 18th World IMACS/MODSIM Congress, Cairns, Australia.

Zhao, W.Y., Chen, Y.N., Li, J.L., Jia, G.S., 2009b. Periodicity of plant yield and its response to precipitation in the steppe desert of the Tianshan Mountains region. J. Arid Environ. 74 (4), 445-449.

Zhou, X.F., Li, Q., Jin, Y., 1994. Studies on forest hydrological cycling. In: Zhou, X (Ed.), Studies on Forest Ecosystems. Northeast Forestry University Press, Harbin, pp. 317-331 (in Chinese).

Zhou, G.Y., Wei, X.H., Luo, Y, Zhang, M.F. Li, Y.L, Qiao, Y.N., Liu, H.G., Wang, C.L, 2010. Forest recovery and river discharge at the regional scale of Guangdong Province, China. Water Resour. Res. 46, W09503. doi:10.1029/2009 WR008829. 